\title{
A Blade Element Theory Based Actuator Disk Methodology for Modeling of Fan Engines in RANS Simulations
}

\author{
Spinner, Sebastian* and Keller, Dennis ${ }^{\dagger}$ \\ DLR Institute of Aerodynamics and Flow Technology, Braunschweig, Germany \\ Schnell, Rainer ${ }^{\star}$ and Trost, Marco ${ }^{\S}$ \\ DLR Institute of Propulsion Technology, Köln, Germany
}

\begin{abstract}
A modified actuator disk method based on blade element momentum theory is applied to an UHBR turbofan engine with the DLR TAU Code. The method is compared against RANS simulations with a common thermodynamic engine boundary condition and high-fidelity $360^{\circ}$ uRANS simulations as well as RANS mixing-plane data from the design process of the engine conducted with the DLR flow solver TRACE. The input data of the actuator disk model comprise sectional lift and drag coefficients of the rotor and stator blades which are obtained by 2D RANS computations of the blade sections performed with the TAU Code. The boundary conditions for these 2D computations are derived from the aforementioned RANS mixing-plane results obtained during the design process of the investigated engine.

Good agreement between the RANS actuator disk method and the uRANS results as well as the RANS mixing-plane data is observed. The actuator disk model is able to predict global engine performance data for different engine operating points. Detailed analysis shows that the actuator disk model is able to reproduce characteristic non-uniform inflow phenomena that could in the past only be modeled with uRANS computations. Additionally the model is capable to accurately predict the thrust distribution between rotor and stator. Therefore it is able to fill the gap between the currently used thermodynamic engine boundary condition and high-fidelity uRANS computations with only a minor increase in required computational resources.
\end{abstract}

\section{Nomenclature}
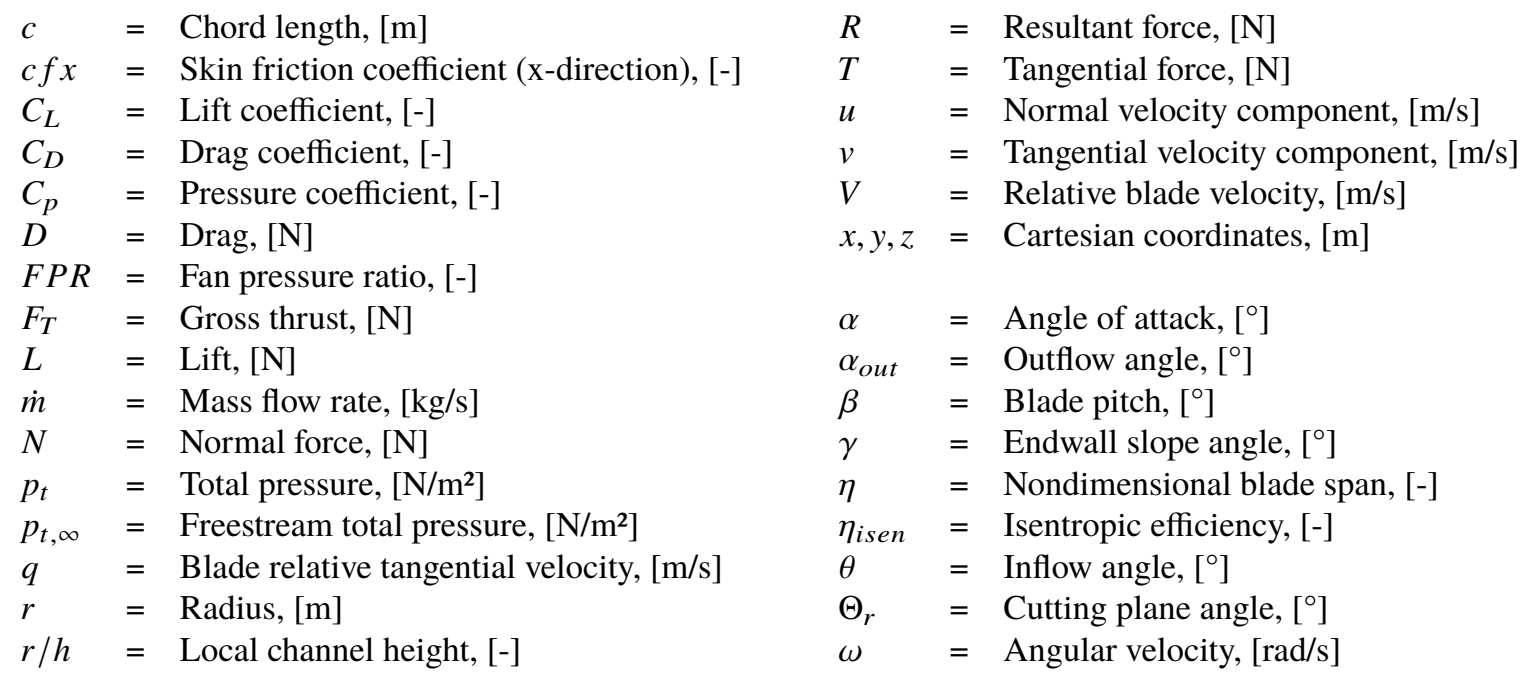

\footnotetext{
* Research Scientist, Transport Aircraft Branch

${ }^{\dagger}$ Research Scientist, Transport Aircraft Branch

${ }^{\ddagger}$ Research Scientist, Fan and Compressor Branch

$\S_{\text {Research Scientist, Fan and Compressor Branch }}$
} 


\section{Introduction}

New high quality engine models with reasonable demand for computing capacity are required in CFD to cope with emerging challenges in the engine airframe design process. Modern turbofan engine designs follow the trend towards larger bypass ratios and lower fan pressure ratios with the target to increase propulsive efficiency. This demands for short nacelle intake designs to limit the additional weight and nacelle drag of the engine. As a result the flow entering the fan is much more susceptible to distortions. This also applies to concepts currently under increased investigation like boundary layer ingestion (BLI). With BLI the close coupling of engine and airframe results in a distorted inflow for the fan as well. Thus future engines are required to perform reliably with non-uniform inflow conditions. As a result of these developments a clear separation of the engine and the airframe during the aerodynamic design is no longer representative. The expected increase in complexity of fan-airframe interaction on future aircraft therefore requires appropriate tools to investigate the mutual aerodynamic interference.

Currently fan engines in the TAU Code are modeled by a boundary condition which is based on a simple thermodynamic model of the engine that is not able to account for non-uniform inflow conditions. A more detailed analysis of engine aerodynamics is available by performing unsteady simulations of the engine taking into account the blade geometry and blade rotation inside the engine. However these simulations are very time and cost intensive due to their large demand for computational resources.

In this work a modified actuator disk (AD) model based on blade element momentum theory (BEMT) is applied to an isolated UHBR (Ultra-High-Bypass-Ratio) fan engine. The simulations show that the AD model is able to reproduce detailed aerodynamic installation effects in contrast to the simple thermodynamic model with the increase in computational costs compared to the latter being only moderate and substantially below those of an unsteady RANS computation. Therefore the proposed actuator disk methodology fills a gap between the simple thermodynamic boundary condition and unsteady engine simulations within the DLR TAU Code.

Previous applications of an actuator disk model to investigate fan engine aerodynamics can be found in [1]. In their work the authors specified relative outflow angles determined from 3D viscous calculations for the rotor $\mathrm{AD}$ whereas stator $\mathrm{AD}$ was assumed to turn the flow to the axial direction. Additionally they introduced a choke model to account for choking of the blade sections [2]. Further simulations with an euler based CFD code modeling rotor and stator stages by actuator disks were performed by [3]. This AD model was provided with radial distributions of stagnation pressure ratio and isentropic efficiency as well as outflow angles to calculate the resulting flow field. A more recent application of an actuator disk model to the same UHBR engine investigated in this paper can be found in [4]. The model is based on an abacus containing values for pressure and temperature jumps and flow deviation angles extracted from RANS computations of isolated rotor/stator stage computations. The authors further described the possibility to estimate the data for the abacus by the Glauert method but due to accuracy and reliability constraints this approach was not pursued any further.

\section{Test Case and Geometry}

The engine studied in this work is depicted in Fig 1. It was designed within the EU Clean Sky 2 project ASPIRE and is representative of a generic modern UHBR turbofan. The nacelle, which was provided by Airbus, is characterized by a very small length-to-diameter ratio and a droop angle at the air-inlet [4]. The fan stage featuring 16 rotor blades and 36 stator blades was designed by the DLR Institue of Propulsion Technology for application in a geared turbofan engine [5].

Depending on the engine operating point different nozzle geometries with adapted nozzle areas are available. The operating conditions studied in this work are presented in Table 1 and feature the Aerodynamic Design Point (ADP), a Take-Off case (TO) and a Take-Off case with a large sideslip angle (TOSL). The latter results in a flow separation at the

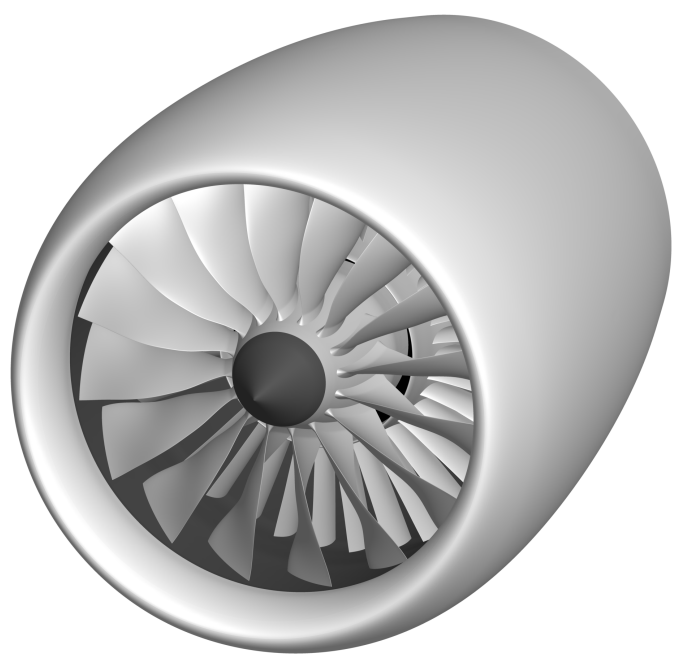

Fig. 1 ASPIRE UHBR turbofan engine. 
lip to enable a test of the boundary conditions for highly distorted inflow conditions. The engine was studied in an isolated configuration. The core engine was not considered in detail and was modeled with the conventional thermodynamic engine boundary condition for all TAU simulations by specifying the massflow and the total temperature ratio from inlet to outlet.

Table 1 Investigated engine operating points for the ASPIRE UHBR test case.

\begin{tabular}{|l|c|c|c|}
\hline & Altitude [ft] & Mach Number [-] & Incidence Angle $\left[{ }^{\circ}\right]$ \\
\hline ADP: Aerodynamic Design Point & 35000 & 0.8 & 3 \\
\hline TO: Take-Off & 700 & 0.27 & 15 \\
\hline TOSL: Take-Off Sideslip & 700 & 0.27 & 33.2 \\
\hline
\end{tabular}

\section{Methodology}

The CFD simulations in this study except for the mixing-plane computations described in Chapter III.D were performed with the DLR TAU Code. The TAU Code [6] is an unstructured finite-volume vertex-based CFD solver developed by DLR. In the following the numerical setup is described briefly. Spatial discretization for the convective fluxes was done using a second order central differencing scheme with scalar dissipation and discretization of the viscous fluxes was done with central differences. The simulations were performed fully turbulent with the Spalart-Allmaras one-equation model [7] used for turbulence modeling. Multigrid methods and local time stepping were used to accelerate convergence. For the unsteady simulations a dual time stepping approach was used.

The computational domain was divided into several blocks which were meshed separately and combined by the code's Chimera method [8]. This procedure allows simulation of relative rotor motion by rotating the rotor chimera block with the implemented motion libraries in the performed uRANS computations. The procedure also permits for the rotor and stator stage blocks to be exchanged for different modeling approaches without the necessity to remesh the nacelle. The blocking strategy is depicted in Fig 2. Additionally chimera surfaces used for postprocessing are marked as follows: in front of the rotor (S1), between rotor and stator (S2), and behind the stator (S3). It should be noted that $\mathrm{S} 2$ is only used in the postprocessing of uRANS and actuator disk simulations. The overall number of points in the meshes were around 28 million for the uRANS simulations, 19 million for the AD simulations and 15 million for the simulations using the conventional TAU engine boundary condition.

\section{A. TAU uRANS Computations}

The procedure of unsteady RANS computations for the ASPIRE UHBR engine with the DLR TAU-Code was already presented in [9] with detailed information on mesh generation, impact of temporal resolution, and mesh convergence together with a detailed analysis of the aerodynamics. For further information the reader is referred to this publication.

In the following only a short overview is given. The simulations were initialized from steady computations without rotation of the rotor block to allow the flow around the nacelle to develop and in turn to accelerate the convergence during the unsteady phase of the simulation. Subsequently the rotor rotation was activated with a very coarse temporal resolution which was refined for the following rotations up to 1152 timesteps per revolution. The results were then time averaged over a quarter rotation which allowed four rotor blades to rotate past nine stator blades. This was deemed sufficient to get a time average of the flow field which was then used for comparison with the actuator disk model and the conventional engine boundary condition.

\section{B. TAU RANS Thermodynamic Engine Boundary Condition}

The engine boundary condition (EBC) within the DLR TAU Code is based on a simple thermodynamic model of the engine in which a total temperature ratio and a total pressure ratio between inlet and outlet planes is set. Additionally the specification of a massflow is allowed which is implemented as an iterative loop adapting the total pressure ratio until the desired massflow through the engine planes is reached. The total temperature ratio is not adapted in this case. Within this work the specification of the massflow is used leaving the total pressure ratio as a free variable to be determined by the model during the simulation. 

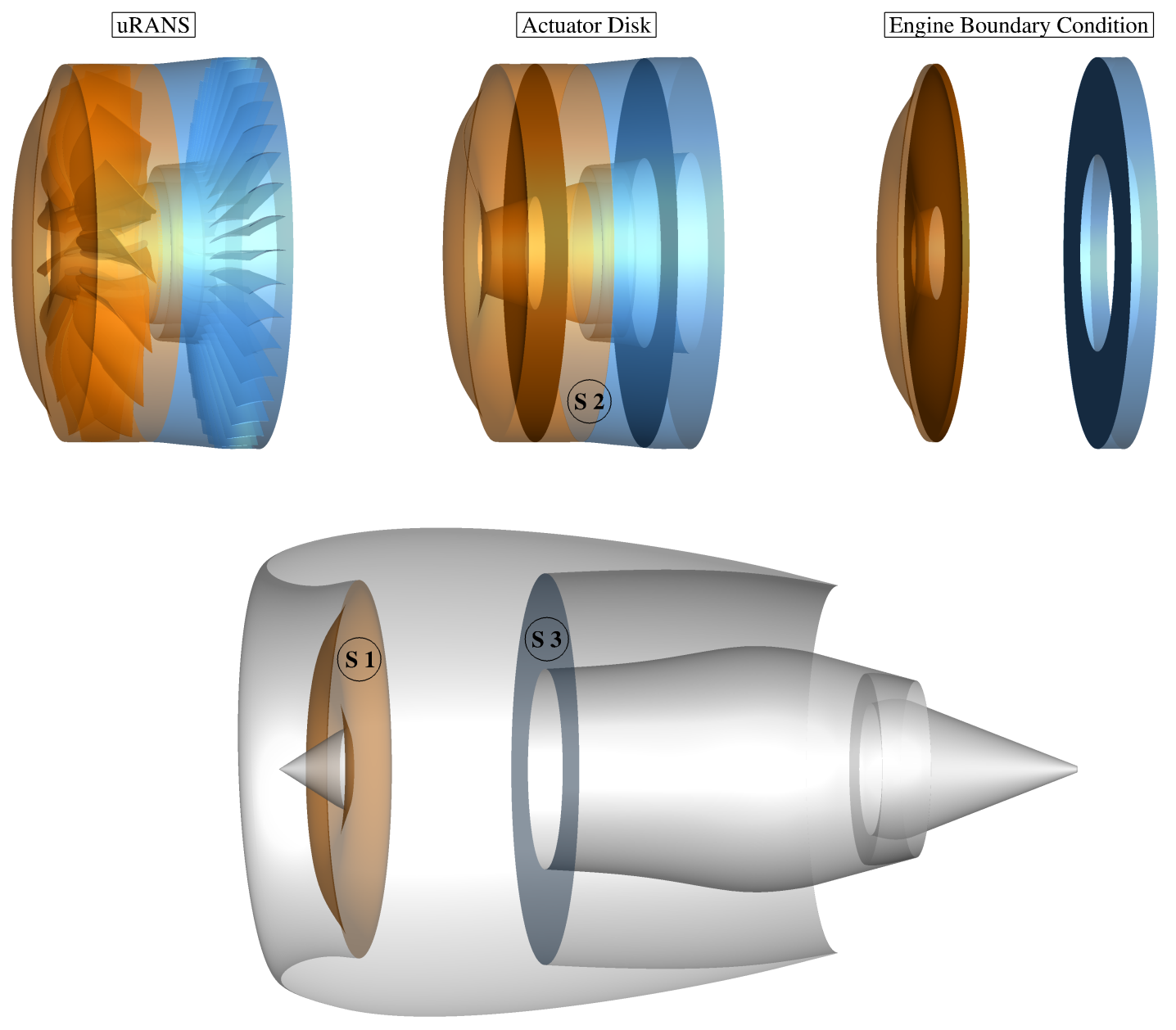

Fig. 2 Mesh blocking strategy for the ASPIRE test case.

\section{TAU RANS Actuator Disk Methodology}

The concept of an actuator disk dates back to propeller theory in the 19th century and is based on work by Froude [10] and Rankine [11]. In the model the blades are replaced by a disk of zero thickness which imposes a pressure jump to the flow whereas the velocity remains continous. In the TAU Code implementation the actuator disk is slightly modified so that there is a discontinious increase in momentum and energy of the flow with the fluxes through the disk being conservative. This enables the embedding into the finite volume discretization of the cell-vertex based flow solver. Further details concerning the implementation of the method can be found in [12] and [13]. Applications of the TAU AD model for propeller aircraft configurations are described in [14-16].

In the $\mathrm{AD}$ model, the force of every blade is integrated in circumferential direction resulting in a rotating line force distribution for every blade. By time averaging these rotating line force distributions, a stationary surface force is obtained which imposes the same momentum per period of revolution to the flow as the blades.

The previously mentioned line force distributions are calculated based on blade element momentum theory. The input data for the model are composed of a parametric description of the blade and radial distributions of sectional lift and drag coefficients for a range of angles of attack. The data are provided as tables which contain the data at discrete radial positions. In between these radial sections the data are interpolated linearly. Figure 3 depicts the geometric relations at the blade section. During each solver cycle the local inflow angle $\theta$ is evaluated and together with the local blade pitch $\beta$ and the specified rotational velocity $\omega$ a resulting angle of attack $\alpha$ is calculated. With this angle corresponding aerodynamic values for $C_{L}$ and $C_{D}$ are extracted from the interpolated input tables allowing for the calculation of local lift $L$ and drag $D$. The resulting force $R$ can then be imposed to the flow as a normal force $N$ 
contributing to the thrust and a tangential force $T$ which produces the swirl.

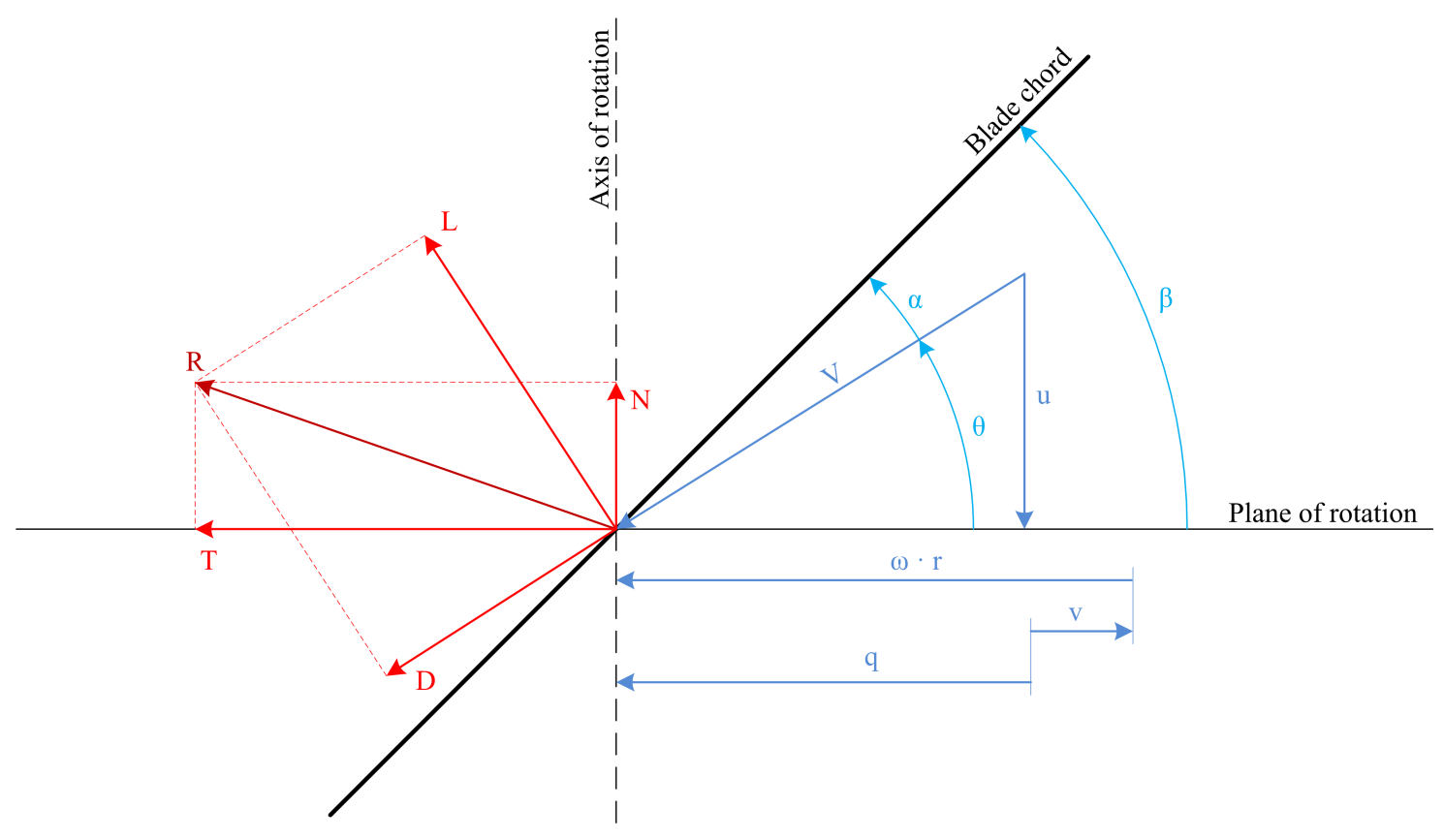

Fig. 3 Geometric relations at the blade section for the BEMT according to [13].

In the BEMT for the propeller, induced velocities in axial and circumferential direction have to be accounted for as described by Glauert [17]. These induced velocities are related to the assumption of a propeller vortex system. The AD model in TAU is directly coupled to the flow field and as a consequence axial induced velocity are considered inherently. In circumferential direction the induced velocities were calculated implicitly by a newton method. Preliminary results suggested that the assumption of a propeller vortex system does not hold for a turbofan engine and the implicit calculation of induced velocities in circumferential direction has to be removed from the code in order to get accurate results with the actuator disk method. All AD simulations in this work where therefore executed without implicitly calculating the induced velocities in circumferential direction.

\section{TRACE RANS Mixing-Plane Computations}

The DLR TRACE code is in continuous development since the beginning of the 1990's and is being extensively used likewise by german national industry and academia. It has been developed predominantly, but not exclusively, for internal and turbomachinery applications. A number of studies in the past dealt with the design and analysis of low pressure ratio fans and their respective integration, as well as the design optimization of fans and open rotors e.g. [18-20]. For the fan design and subsequent analysis of the entire intake and fan system, different simulation strategies available in TRACE were applied: a RANS based approach using classical single passage simulations with a mixing-plane (MP) approach between rotor and stator was used during the many design iterations, allowing for an efficient performance assessment of the fan stage over the entire flight envelope; results taking advantage of this approach are the basis for the comparisons as presented in this paper. During the further analysis of the entire fan and intake system as reported in [21] and [22], a uRANS based, single passage approach in time domain using time-shifted boundary conditions was used [23]; a potentially more efficient approach for these kind of simulations comprised of a non-linear approach formulated in frequency domain method and referred to as harmonic balance (HB) [24]. Both methods have in the past demonstrated to be well suited for this kind of application, with the HB method allowing for results to be obtained overnight. All mentioned CFD techniques share the same code basis, are formulated based on finite volumes and take advantage of identical and Roe-type flux formulations with 2nd order MUSCL interpolation to the cell vertices. The solver methodology is implicit with second order temporal accuracy and the Menter SST k- $\omega$ turbulence model being applied predominantly. Blade row coupling was realized either with classical mixing-planes, formulated in a non-reflecting manner, or with a conservative zonal approach, also yielding 2nd order of accuracy. A combination of varying mesh fidelities in the different regions of the computational domain was used, with the number 
of cells used as summarized in Table 2 , mesh convergence studies as well as turbulence model sensitivity studies were carried out prior to commencing with the fan stage design.

Table 2 DLR TRACE structured grid sizes (M: million of nodes).

\begin{tabular}{|c|c|c|c|}
\hline & Intake & Rotor & Stator \\
\hline RANS/uRANS/HB 360 & $12 \mathrm{M}$ & $16 \times 1.5 \mathrm{M}=24 \mathrm{M}$ & $36 \times 0.5 \mathrm{M}=18 \mathrm{M}$ \\
\hline
\end{tabular}

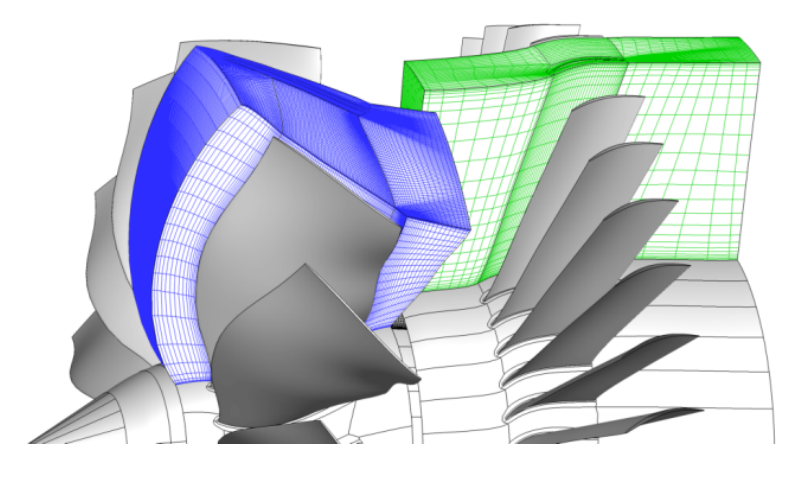

Fig. 4 DLR TRACE RANS based single passage setup using a mixing-plane approach [21].

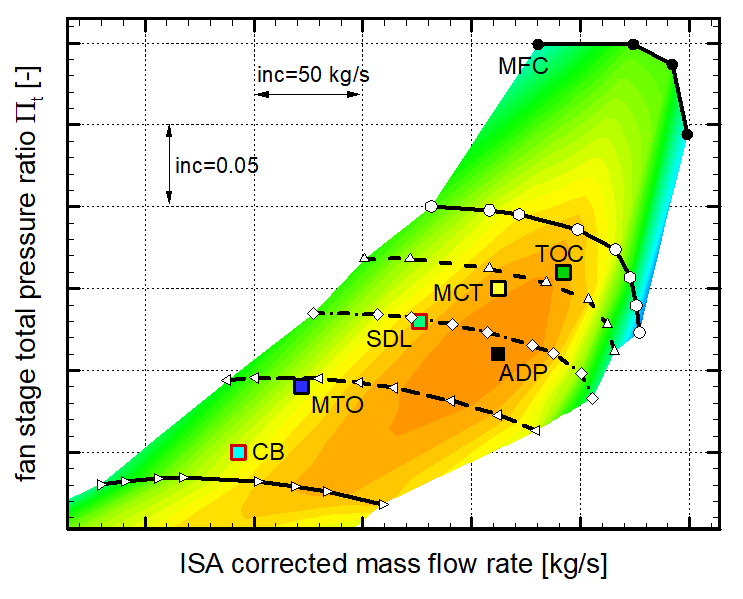

Fig. 5 DLR TRACE RANS-based fan performance characteristics with efficiency contour levels [21].

\section{Actuator Disk Input Data Generation}

The chosen actuator disk approach bases on sectional data of the fan blades that replicate their geometric and aerodynamic properties. For the evaluation of the aerodynamic properties, sectional airfoil geometries are extracted from the blades considering the local taper angles of the annular channel. Therefore, the angle of the cutting plane is calculated based on the difference of the slope angles at the tip and the hub, which is then multiplied by the relative radial position of the airfoil that is to be extracted (Eq1).

$$
\Theta_{r}=\gamma_{h u b}+\left(\gamma_{t i p}-\gamma_{h u b}\right) *\left(r-r_{h u b}\right) /\left(r_{t i p}-r_{h u b}\right)
$$

For the sake of simplicity, the airfoil geometries were extracted by planar surfaces instead of conical surfaces. The latter may have an additional beneficial impact on the accuracy of the sectional aerodynamic data. After extracting the airfoil geometries, the aerodynamic data can be collected by various approaches such as $2 \mathrm{D}$ panel methods or higher fidelity methods like 2D RANS simulations. In contrast to the estimation of airfoil data for propellers, the assumption of the blades operating under freestream conditions is not sufficiently adequate for the estimation of the fan/stator blade performance. Additional to the typical parameters that have to be considered such as Reynolds number and Mach number, stagger effects of the blade cascade are obviously important. Moreover, the pre-entry and post-exit static pressure conditions have been found to be the most significant parameters that have to be considered. Considering these effects by simulating a simplified 3D geometric model of a blade section with tapered channel and a following stator cascade (for rotor airfoils) or preceding rotor cascade (for stator airfoils) showed promising results. The most simplistic way however to consider these effects is to compute a 2D airfoil with periodic boundary conditions below and above the airfoil and to imply the streamwise static pressure evolution within the annular channel via inlet and outlet boundary conditions. Typically, the required data can be extracted from the design process of the fan stage, e.g. from streamline curvature methods or (steady) RANS simulations. Figure 6illustrates the basic setup of the boundary conditions for the evaluation of the aerodynamic blade properties via 2D RANS computations as used in the present investigation. 
The upper and lower boundary conditions (blue lines) of the computational domain are set to periodic ones in order to simulate the cascade effect while considering the local cascade pitch and stagger angle. The inlet boundary condition (green line) is a farfield one with the flow properties derived from the design process. At the outlet (red line), a constant pressure condition is imposed with the static pressure value again being extracted from the design process.

inlet: farfield BC

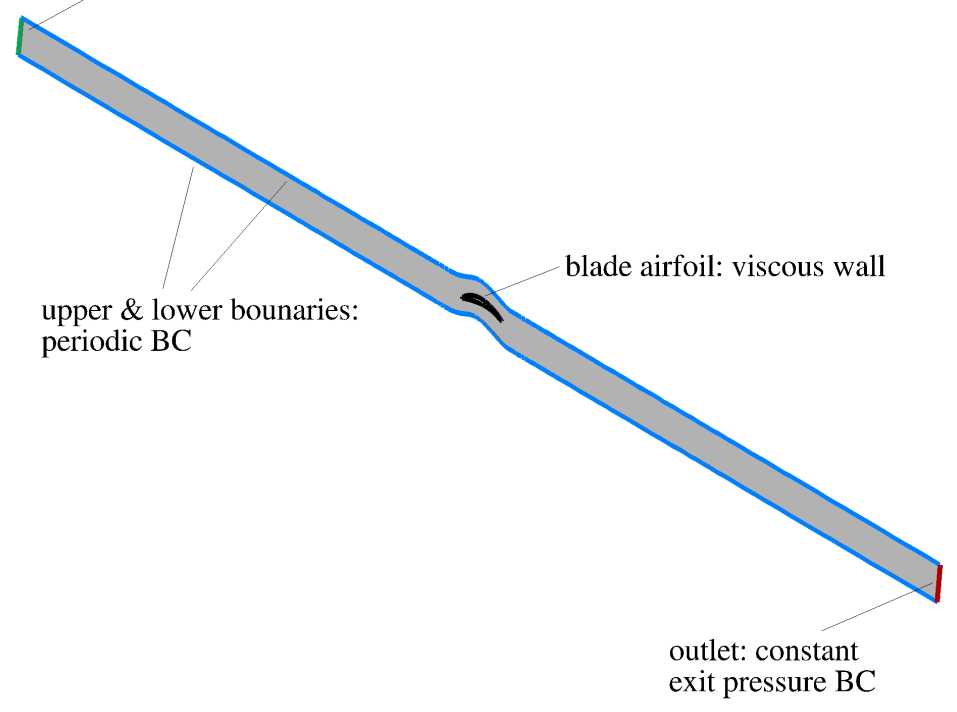

Fig. 6 Setup of boundary conditions for 2D computations.

Figure 7 compares the distributions of the surface pressure coefficient from 2D rotor airfoil computations with varying boundary conditions to the ones of the 3D uRANS computation at ADP conditions. In the 3D uRANS computation several factors, such as the the induced angle of attack, swirl, and the effective onset flow velocity, affect the local incidence angle. As the aim of this comparison is to investigate whether the 2D computations can generally reproduce the basic aerodynamic behavior of the rotor, the $C_{p}$ distributions of the $2 \mathrm{D}$ computations are extracted at the angle of attack at which the suction peak of the 2D computation with boundary conditions extracted from the uRANS computation (green line) fits best to the one of the 3D uRANS computation (black line). When neither considering the pressure evolution within the annular channel nor the cascade effects (orange line), the $C_{p}$ distribution differs completely from the one extracted from the 3D uRANS computation (black line), as shown in Fig. 7a for $\eta=0.5$. The suction peak is exaggerated, whereas the stagnation pressure at the trailing edge is too low. Furthermore, the distribution indicates a double shock on the suction side that is not present in the uRANS computation. When the cascade effects are considered whereas the static pressure evolution within the annular channel is not (red line), the suction peak moves onto the pressure side of the airfoil. Downstream of the suction peak, the pressure recovers to a stagnation pressure at the trailing edge, which is still too low compared to the one from the uRANS computation. On the suction side, the $C_{p}$ distribution indicates one shock. Considering the cascade effects via periodic boundary conditions and the static pressure evolution within the annular channel based on data extracted from the RANS computations with TRACE (blue line), the $C_{p}$ distribution of the 2D airfoil computation generally agrees well with the one from the uRANS computation. The stagnation pressure at the leading edge as well as at the trailing edge match within acceptable accuracy. The suction peak inherently agrees well due to the process of comparison. The most notable differences in the $C_{p}$ distribution can be observed for $0.1 \leq X / c \leq 0.8$, where the surface pressure appears to be too high compared to the uRANS computation. Extracting data for the inlet and outlet boundary conditions from the uRANS computation instead of using design data does not improve the $C_{p}$ distribution substantially. This leads to the conclusion that the remaining differences between the $C_{p}$ distribution from the 2D airfoil computation and the one from the uRANS are rather caused by $3 \mathrm{D}$ effects than by inaccurate boundary condition data.

Figure $7 \mathrm{~b}$ compares the $C_{p}$ distributions at $\eta=0.25$. Here, the $C_{p}$ distributions of the $2 \mathrm{D}$ computations with cascade effects and static pressure evolution within the annular channel being considered (blue and green lines) still reproduce the basic characteristics of the distribution from the 3D uRANS computation. However, notable differences can be observed for $X / c \leq 0.5$ on the suction side and for $X / c \geq 0.4$ on the pressure side. At $\eta=0.75$ (Fig. 7c), the $C_{p}$ 


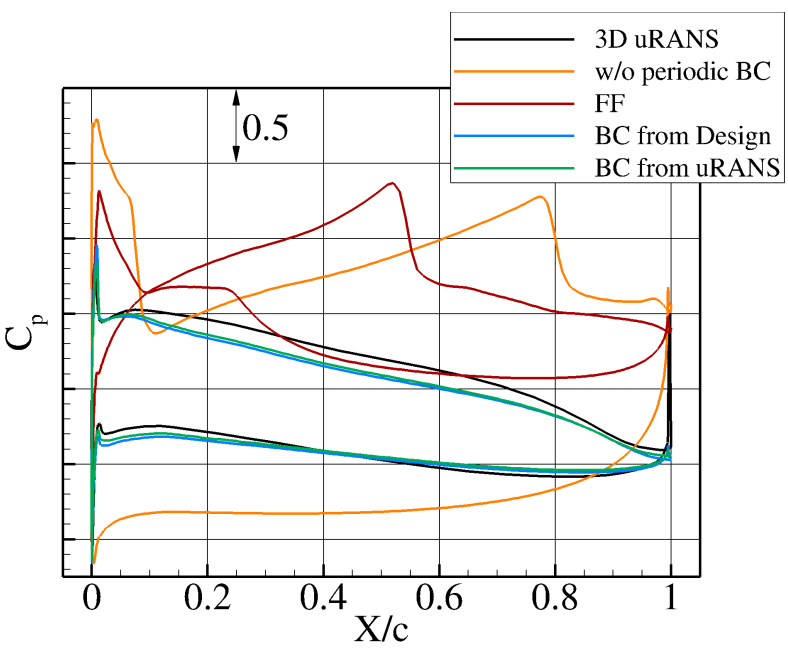

(a) $\eta=0.5$

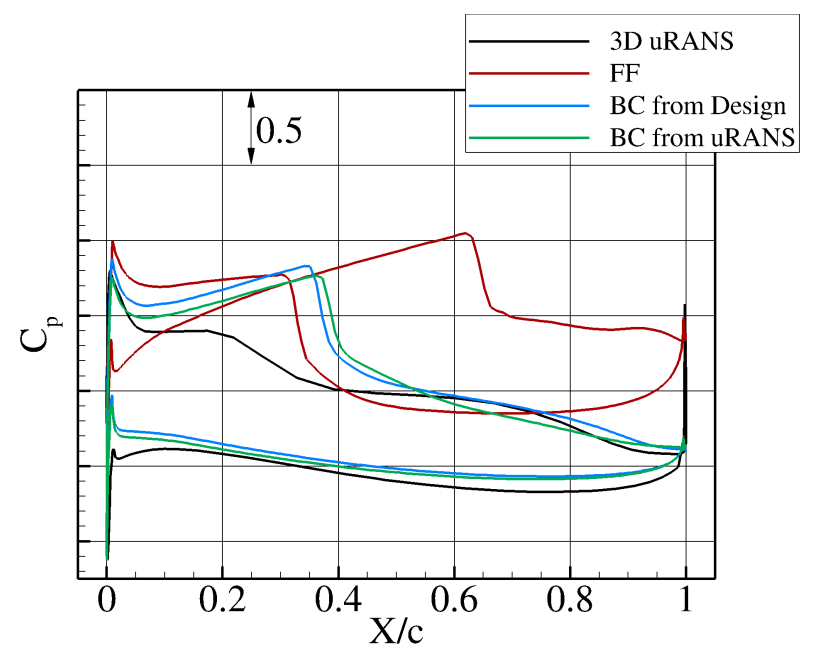

(c) $\eta=0.75$

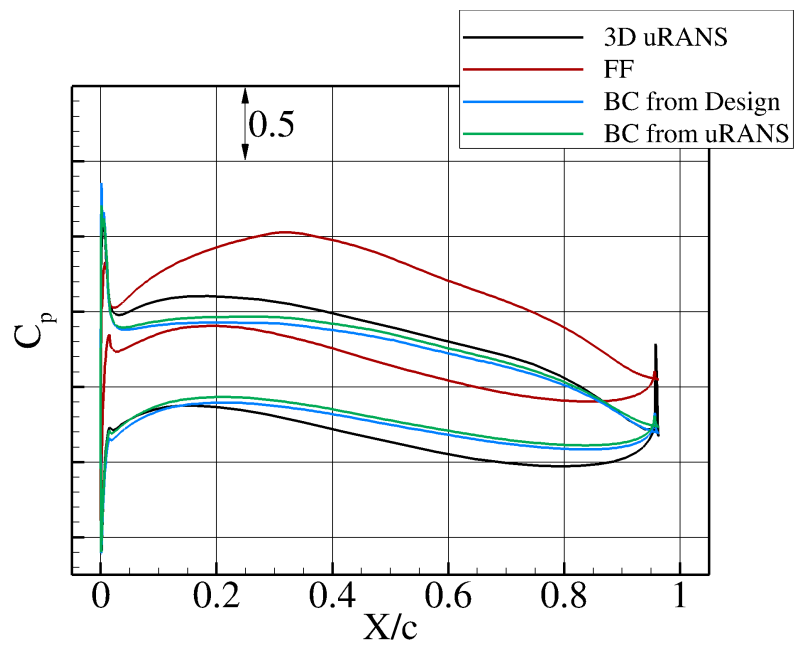

(b) $\eta=0.25$

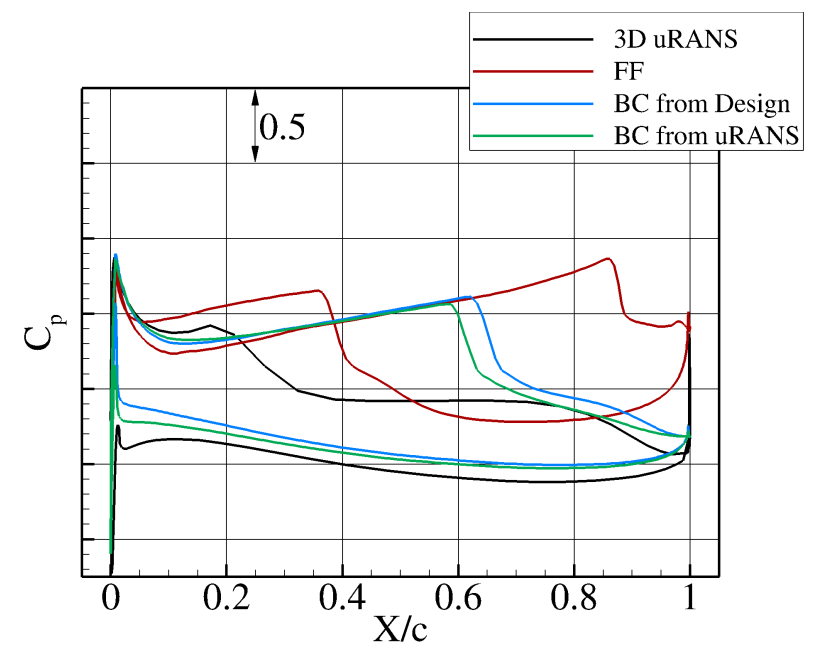

(d) $\eta=0.90$

Fig. 7 Impact of domain boundary conditions on sectional distribution of the surface pressure coefficient (rotor).

distributions of the 2D computations (with cascade effects and static pressure evolution being considered) indicate a reacceleration of the flow after an initial deceleration downstream of the suction peak. A shock at $X / c \approx 0.4$ abruptly ends the flow acceleration. In contrast the distribution from the 3D uRANS computation indicates a steadier pressure recovery. At $\eta=0.9$ (Fig. 7d, the differences in the $C_{p}$ distribution between the 2D computations and the 3D uRANS computation increase as the shock in the 2D computations moves towards the trailing edge.

Analogous to Fig. 7. Fig. 8 compares the $C_{p}$ distributions of the stator for three different sections. For all sections, the $C_{p}$ distributions of the $2 \mathrm{D}$ computations that consider only the cascade effects but do not consider the annular channel pressure evolution differ substantially from the ones of the 3D uRANS computation. In contrast, the $C_{p}$ distributions of the 2D computations that consider both effects resemble the ones of the 3D uRANS computation. All of these $2 \mathrm{D}$ computations however yield a lower surface pressure between $0.1 \leq X / c \leq 0.8$ on the suction side. A notable difference can also be observed between the case based on the design data and the one based on the 3D uRANS data for $\eta=0.25$. Here, the stagnation pressure coefficient at the trailing edge of the 2D computation with design data appears higher compared to the ones of the 3D uRANS computation and the 2D computation based on 3D uRANS data. Skin friction lines on the stator of the 3D uRANS computation indicate considerable flow separation here. 


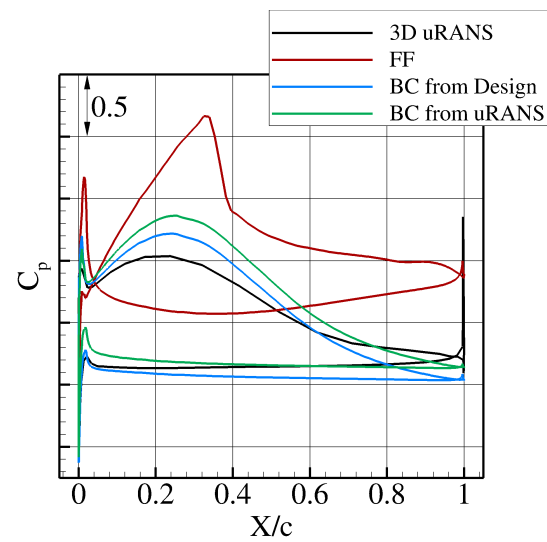

(a) $\eta=0.25$

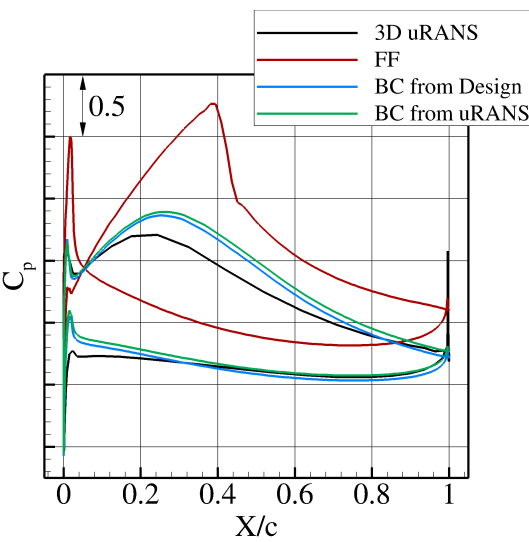

(b) $\eta=0.5$

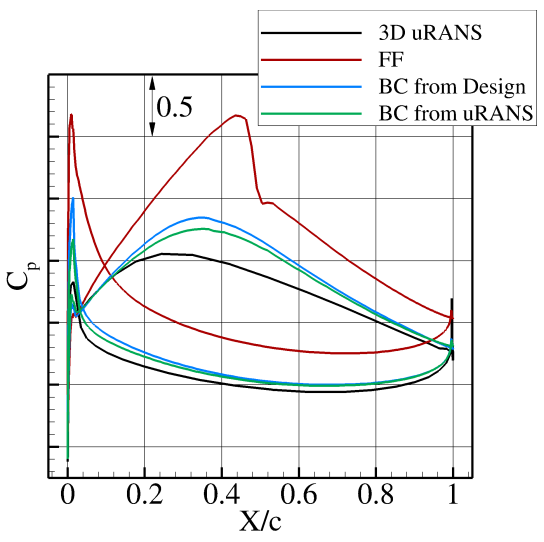

(c) $\eta=0.75$

Fig. 8 Impact of domain boundary conditions on sectional distribution of the surface pressure coefficient (stator).

In the present investigation the aerodynamic airfoil dataset based on boundary conditions extracted from the design process is used for the RANS computations with actuator disk. While the described approach of estimating the sectional aerodynamic data for the rotor and the stator does not reflect 3D effects such as secondary flow, boundary layer centrifugation, tip clearance vorticity, and wall boundary layers, it still gives an acceptable approximation for the most part of the blades. The discrepancies however increase towards the blade tip as 3D effects begin to play an increasingly important role.

\section{Results}

In this section results are analyzed for all operating points investigated. Data obtained from the uRANS simulation are taken as reference and results from the steady AD and engine boundary condition simulations are compared against it. The airfoil dataset for the AD model was generated based on boundary conditions from the design process according to chapter IV for the ADP case and used throughout all other operating points as well. The computational effort for the actuator disk simulations was around 15\% - 25\% higher compared to the simulations with the conventional engine boundary condition and at least an order of magnitude less than for the uRANS computations.

\section{A. Aerodynamic Design Point}

A qualitative assessment for the aerodynamic design point allows to show some distinct features of the AD model. Figure 9 pictures normalized total pressure distributions on a horizontal slice through the engine including streamlines for all three modeling approaches with TAU. As can be seen in Fig. 9c the engine boundary condition only imposes a uniform total pressure rise and no swirl to the flow. Contrary to this the AD model in Fig. $9 \mathrm{~b}$ imposes a swirl to the flow at the position of the rotor disk and removes this swirl again at the stator disk similarly to what can be observed in the uRANS computation in Fig. 9a. Additionally the total pressure rise imposed by the actuator disk is not uniform but highly sensitive to the inflow conditions. The effect of asymmetric load distribution due to the short intake and the engine incidence angle of $3^{\circ}$ can be observed for the uRANS computation as well as for the actuator disk. Due to the inclination of the engine the blades moving in downward direction experience a locally increased angle of attack while the blades moving in upward direction experience a locally decreased angle of attack (direction of rotation counterclockwise viewed from the front). This effect results in an increased total pressure rise on the downward moving side and a decreased total pressure rise on the upward moving side which can be observed both in Fig. 9a and 9b.

Pitch averaged radial distributions were computed on the chimera plane aft of the stator section (see Fig. 2) to allow for a quantitative assessment. Figure 10 shows pitch averaged radial distributions of total pressure ratio and isentropic efficiency for the three engine modeling approaches in TAU and in addition the RANS mixing-plane simulation carried out with TRACE. Once again the uniform distribution of flow variables of the engine boundary condition can be observed. The pressure ratio in the mixing-plane simulation is generally increased compared to the uRANS simulation 


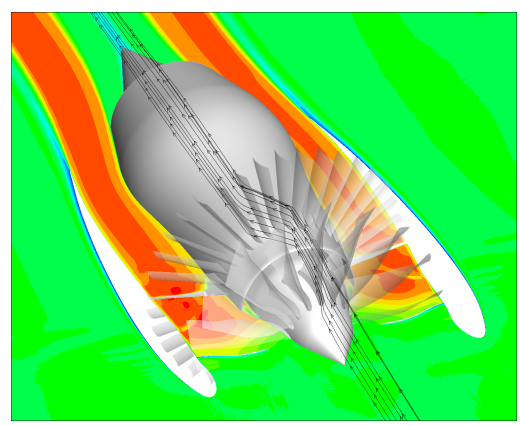

(a) URANS

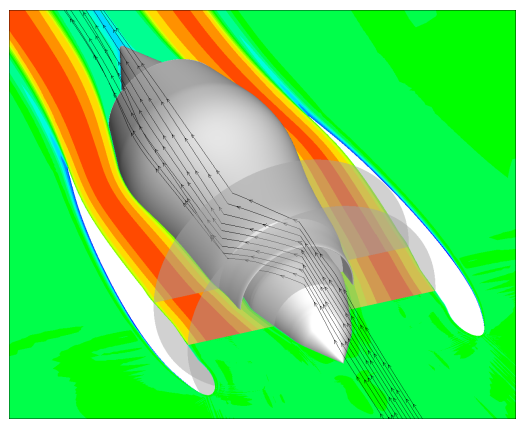

(b) RANS Actuator Disk

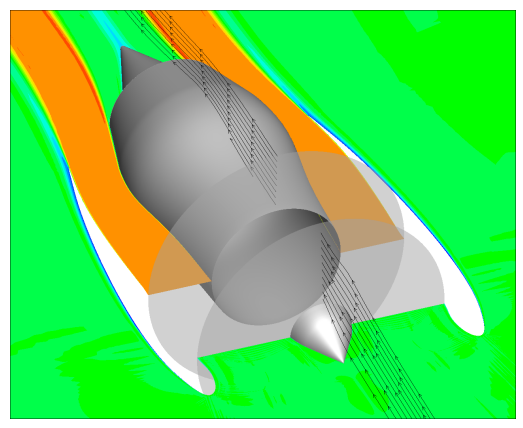

(c) RANS Engine BC

Fig. 9 Normalized total pressure distribution in engine plane for different engine modeling methodologies (ADP).

whereas the shape of the radial distribution agrees well except for the hub region close to the wall. The higher total pressure ratio in the mixing-plane simulation results from a shift of the operating point during the design phase of the engine whereby the fan speed was adapted on a small scale. The actuator disk data agree very well with the uRANS data for the total pressure ratio in the midspan region in Fig. 10a whereas in regions closer to the shroud the total pressure ratio of the actuator disk model is significantly lower compared to the uRANS simulation. This is consistent with the deviations observed for the pressure distributions in Fig. 7 indicating that additional 3D effects play an important role in proximity to the tip region.

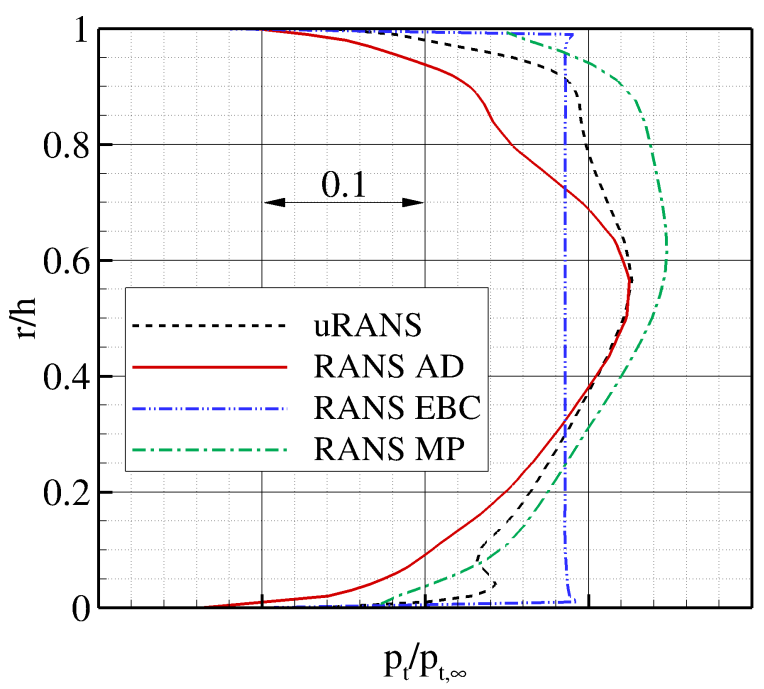

(a) Total Pressure Ratio

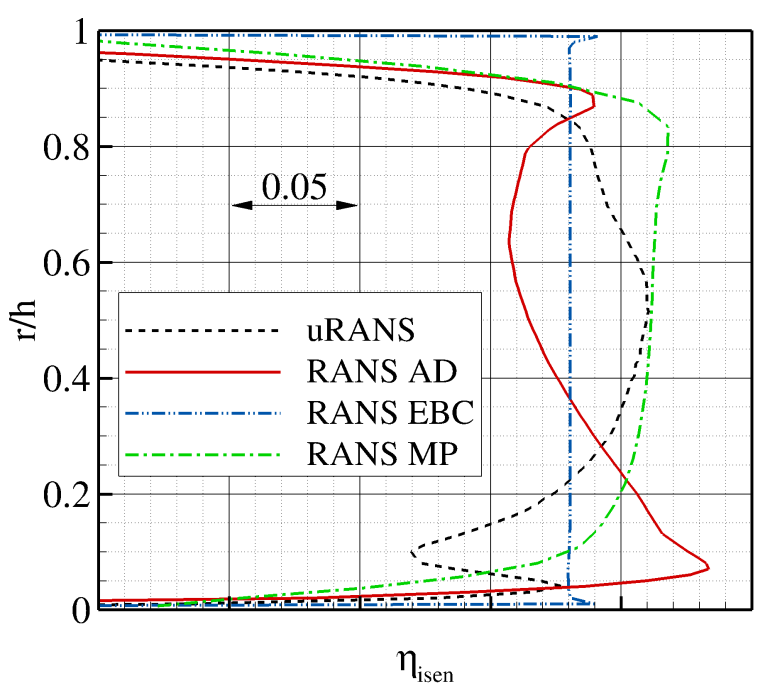

(b) Isentropic Efficiency

Fig. 10 Pitch averaged radial distributions in the bypass duct aft of the stator plane (S3) for the ADP operating point. 
Isentropic efficiency was computed from the total pressure ratio and total temperature ratio data and is shown in Fig. 10b. Again, with the engine boundary condition a constant radial distribution can be seen since this is directly imposed by the model. Comparing uRANS and RANS mixing-plane results very good agreement can be observed in the midspan region whereas the uRANS computation shows deteriorating efficiency in the hub and shroud regions. The actuator disk model is not able to reproduce the radial distribution of isentropic efficiency accurately. Efficiency is overestimated in the hub region whereas it is largely underestimated for the midspan region. Around the tip the efficiency curve recovers and even exceeds the levels of the uRANS simulation but overall the shape of the isentropic efficiency distribution does not match the uRANS data at all.

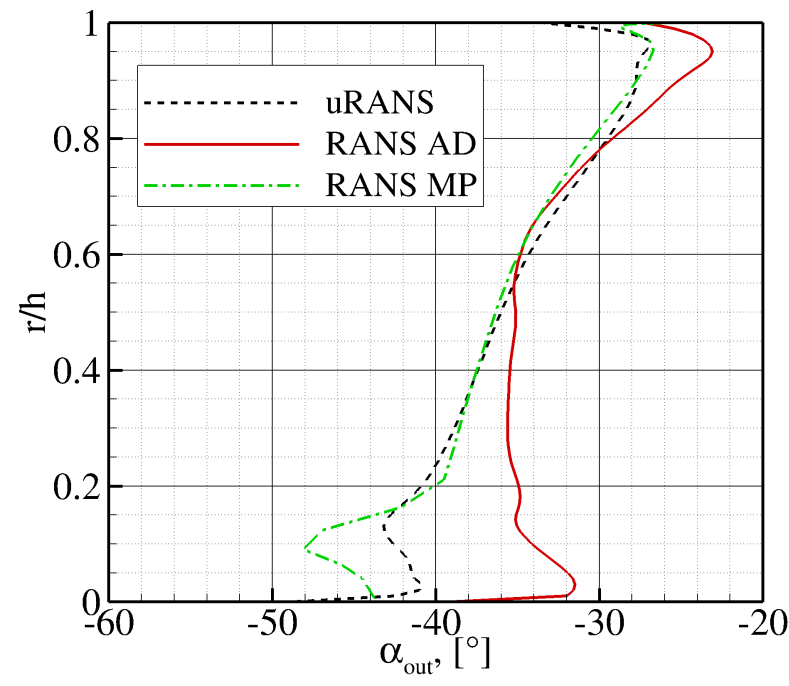

(a) Rotor Exit

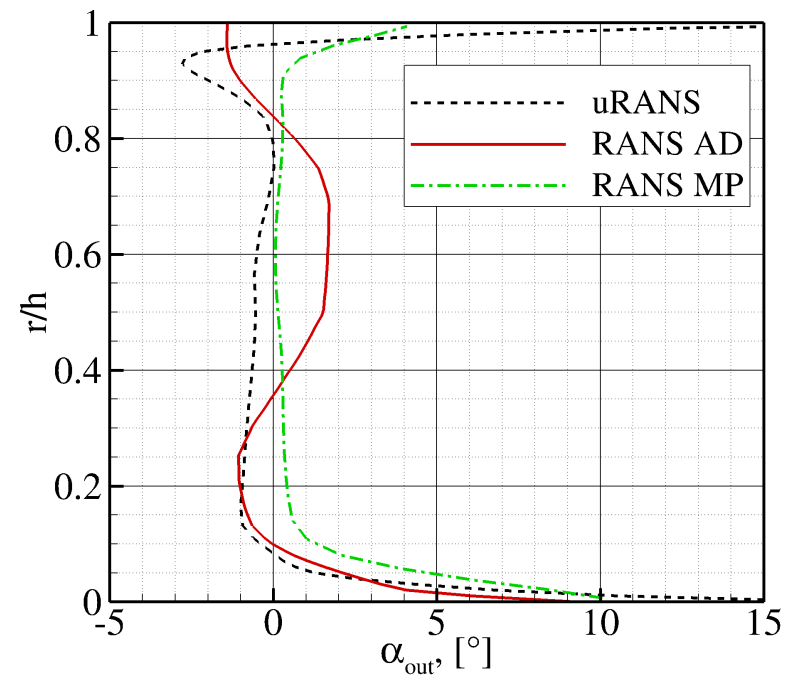

(b) Stator Exit

Fig. 11 Pitch averaged radial distribution of outflow swirl angles for the ADP case.

As already shown in Fig. 9b the AD model allows to account for the swirl imposed by rotor rotation. Figure 11 shows pitch averaged flow deviation angles on the chimera planes aft of the rotor (S2) in Fig. 11a and stator (S3) in Fig. $11 \mathrm{~b}$ The outflow swirl angles behind the rotor in Fig. 11a show very good agreement between uRANS and RANS mixing-plane data except for a small deviation in the hub region. The AD model is able to predict this data accurately in the midspan and, with small deviations, in the tip region. A larger deficit in flow turning can be observed for lower radii closer to the hub. Rotor outflow angles are negative due to counterclockwise rotation of the rotor. The redeflection of the flow towards the axial direction by the stator can be seen in Fig. 11b Due to the fact that the TRACE simulation was included in the engine design process it is close to an outflow angle of $0^{\circ}$ for the most part which was one of the design targets. Although there are some deviations both the uRANS simulation as well as the AD model predict the outflow angle to be below $+/-2^{\circ}$ with the AD model showing the most variation in radial direction. In the midspan region the stator in the AD model is turning the flow to much resulting in a positive outflow angle whereas closer to the channel walls negative outflow angles can be observed indicating insufficient flow turning.

Table 3 Thrust component distribution comparison (ADP).

\begin{tabular}{|l|c|c|}
\hline$\%$ Overall Thrust & uRANS & RANS Actuator Disk \\
\hline Rotor & $62 \%$ & $62 \%$ \\
\hline Stator & $28 \%$ & $26 \%$ \\
\hline Core & $10 \%$ & $12 \%$ \\
\hline
\end{tabular}

An evaluation of the different thrust components in the engine can be seen in Table 3 . Since it is not possible to make such a distinction for the engine boundary condition, only uRANS and AD model data are shown. Thrust data is not available for the TRACE computations. The agreement between the thrust distributions is good. In both computations $60 \%$ of thrust are produced by the rotor stage whereas the stator delivers roughly $28 \%$ in the uRANS 
case and 26\% with the AD model. The contribution of the core engine to the overall thrust is relatively small for both modeling approaches. In this context it should be mentioned that the overall gross thrust produced in the AD simulation was lower compared to the uRANS simulation and as a consequence the portion of the core engine, which was modeled by the conventional thermodynamic boundary condition, is therefore larger.

Table 4 Performance characteristics compared to uRANS data (ADP).

\begin{tabular}{|l|c|c|c|}
\hline$\frac{X-X_{U R A N S}}{X_{U R A N S}}$ & RANS Actuator Disk & RANS Engine BC & RANS Mixing-Plane \\
\hline$\dot{m}$ & $-3.0 \%$ & $-0.7 \%$ & $+2.28 \%$ \\
\hline$F P R$ & $-2.1 \%$ & $-0.2 \%$ & $+1.28 \%$ \\
\hline$\eta_{i s e n}$ & $-0.5 \%$ & $+1.6 \%$ & $+1.72 \%$ \\
\hline$F_{T}$ & $-6.1 \%$ & $+1.7 \%$ & $\mathrm{n} / \mathrm{a}$ \\
\hline
\end{tabular}

In Table 4 global engine performance metrics for the actuator disk approach and the engine boundary condition are compared against the uRANS results. It can be observed that the actuator disk model is able to capture the engine massflow and fan pressure ratio within $3 \%$ of the uRANS results whereas deviations in terms of thrust are a bit larger at around $6 \%$. Isentropic efficiency with the AD model is very close to the uRANS data with a deviation of around $0.5 \%$. In comparison, the engine boundary condition results agree very well with the uRANS results for the massflow and FPR. This was expected since the boundary condition was set to match the massflow by iterating the pressure ratio. The error in terms of thrust is just below $2 \%$ and is therefore also smaller than the error observed with the AD model. Contrary to that the deviation in terms of isentropic efficiency is larger than with the AD model but still comparatively small. With the mixing-plane approach massflow and pressure ratio are slightly higher compared to the uRANS computation due to a shift of the operating point during the engine design phase as already mentioned earlier. As it could already be inferred from the radial distribution in Fig. $10 \mathrm{~b}$ overall isentropic efficiency is explicitly higher with the mixing-plane simulation than within the uRANS simulation.

\section{B. Take-Off}

The Take-Off case is characterized by a high angle of attack of $15^{\circ}$ and a high power setting. Consequently the effect of asymmetric blade loading already discussed for the ADP case is even more clearly visible. Figure 12 shows normalized total pressure distributions on a horizontal slice through the engine viewed from the top. The direction of rotation of the fan is counterclockwise when looking from the left. It can be observed that the AD model as well as the uRANS simulation show a stronger total pressure rise in the upper part of the picture where the local blade incidence angle is increased due to the blades moving in downward direction. The higher total pressure is also convected through the stator stage and still visible in the jet at the nozzle exit for both the AD and the uRANS simulation. Again, no such effect can be modeled by the thermodynamic engine boundary condition.

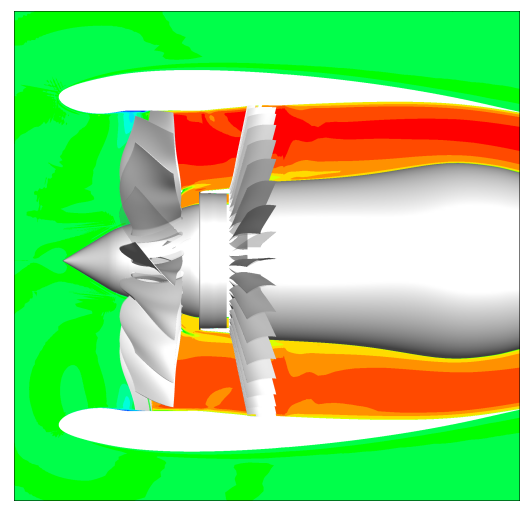

(a) URANS

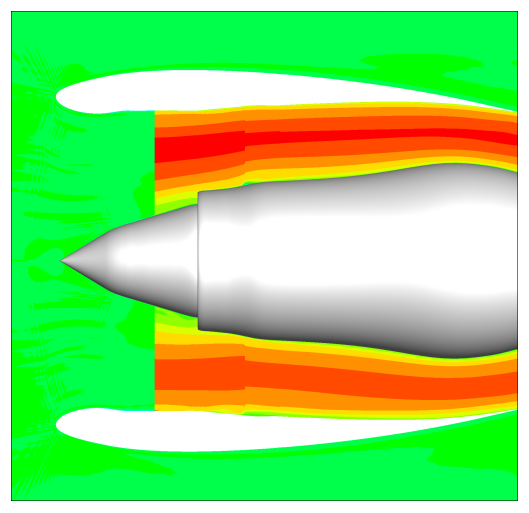

(b) RANS Actuator Disk

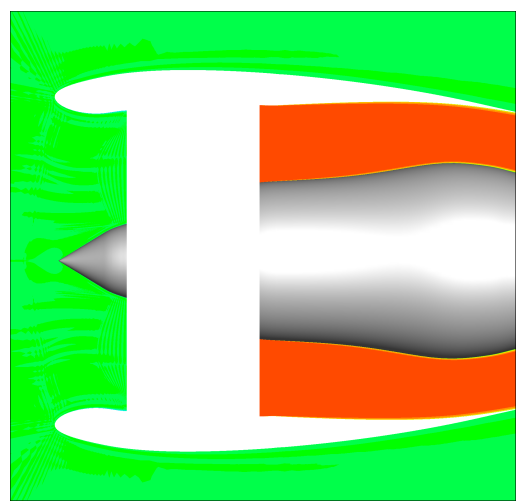

(c) RANS Engine BC

Fig. 12 Normalized total pressure distribution in engine plane for different engine modeling methodologies (TO) (engine not to scale). 
The extent of this phenomenon in the circumferential and radial direction is shown in Fig. 13 Slices of normalized total pressure in the engine nozzle aft of the stator are depicted. The wakes of the stator blades can be seen for the uRANS simulation in Fig. 13a. The overall agreement between the AD model and the uRANS data is good. The maximum of the total pressure distribution can be identified between the 6 and 9 o'clock position for both the uRANS simulation and the $\mathrm{AD}$ simulation whereas the engine boundary condition is as expected not able to reproduce this effect. The radial position of the previously described maximum is shifted to slightly lower radii for the AD model compared to the uRANS simulation. For the AD model a non-uniformity of the total pressure distribution close to the shroud region in the bottom right of Fig. 13b can be observed. This could be traced down to a flow separation in the tip region behind the fan actuator disk which is smeared out radially and circumferentially by the stator AD. The occurrence of this flow separation seems to be linked to the treatment of the forces at the actuator disk close to the viscous wall which will need further investigation. Currently the force at the disk close to wall is scaled to zero to avoid a nonphysical jump in static pressure inside the boundary layer which destabilizes the simulation.

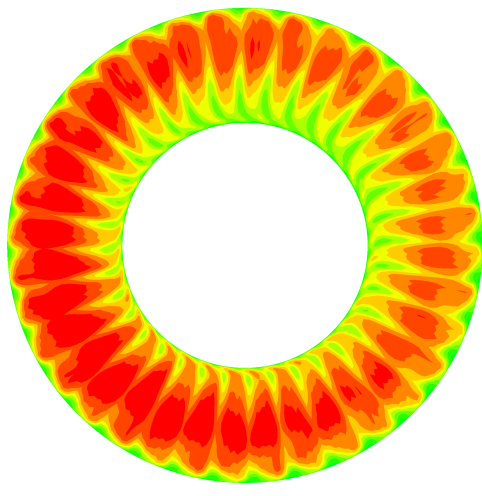

(a) URANS

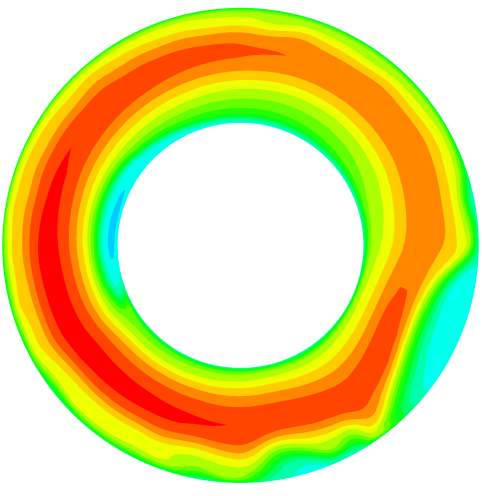

(b) RANS Actuator Disk

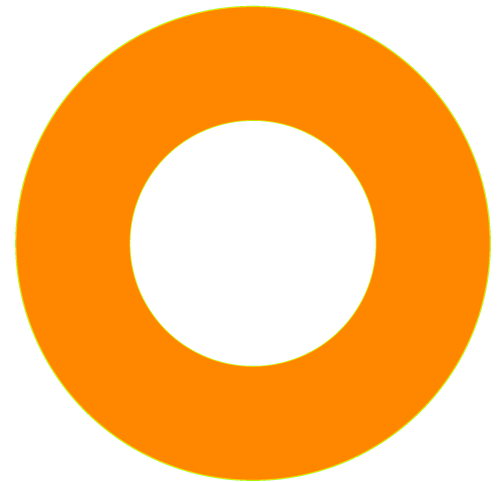

(c) RANS Engine BC

Fig. 13 Normalized total pressure distribution on $x$-Slices aft of the stator for different engine modeling methodologies (TO).

Pitch averaged radial distributions where extracted for the Take-Off case as well. Figure 14 shows those for the total pressure ratio and the isentropic efficiency. For comparison the mixing-plane simulation results are shown as well. The data for the engine boundary condition are shown but not discussed in detail since it does not provide any further understanding due to its one-dimensionality.

The total pressure ratio in Fig. 14a shows very good agreement between the uRANS data and the RANS mixing-plane results. Only in the shroud region the total pressure distribution of the uRANS simulation is slightly decreased compared to the mixing-plane data. Looking at the AD model the agreement is very good in the lower half of the channel but a significant lack of total pressure in the region closer to the shroud is visible. Nevertheless the radial position of the maximum total pressure is predicted with high accuracy by the AD model and agrees very well with the uRANS and mixing-plane data. In addition, despite the weaker course in the upper range, the AD model shows a weak inflection point in total pressure ratio at approximately $80 \%$ channel height as can be seen in the uRANS data as well.

For the isentropic efficiency distribution in Fig. 14b the agreement between the uRANS data and the mixing-plane simulation is good with moderate deviations in the hub and shroud region which are comparable to what was observed for the ADP case. Again the AD model is not able to correctly predict the isentropic efficiency distribution. Efficiency levels in the hub region are overestimated using the actuator disk whereas in the midspan and shroud region isentropic efficiency is substantially below the uRANS and mixing-plane simulation distributions. Contrary to what was observed for the ADP case no recovery of isentropic efficiency levels in the tip region is visible which results probably from pitch averaging over the flow separation in the tip region discussed for Fig. 13b

The evaluation of the different thrust components in the engine is shown in Table 5 for uRANS and AD model results. Thrust data was not available for the mixing-plane computations. Columns may not add up to $100 \%$ due to roundup errors. The agreement between the thrust distributions is good despite the flow separation. In both the uRANS computation and the AD modeling $60 \%$ of overall thrust are produced by the rotor stage whereas the stator delivers $28 \%$ and $27 \%$ respectively. The contribution of the core engine to the overall thrust is slightly larger for this operating point. 


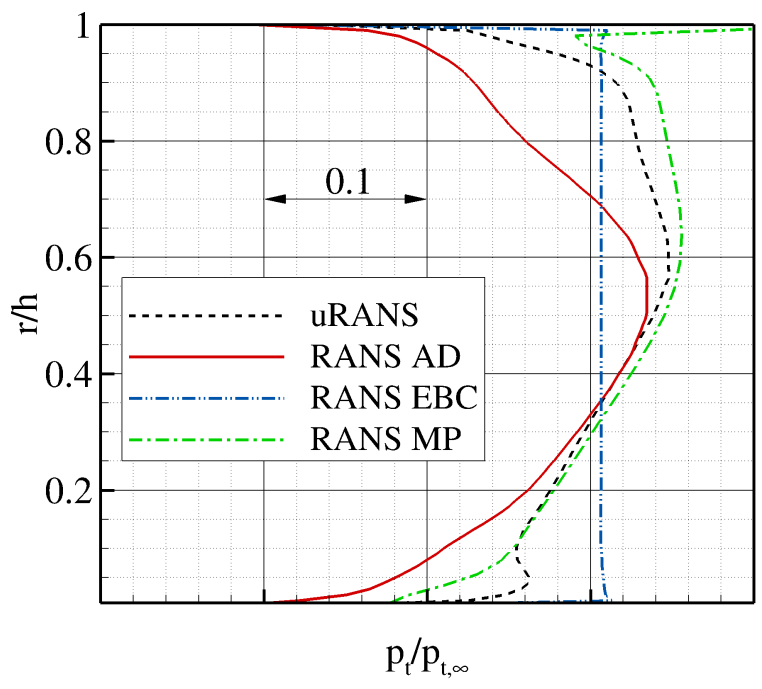

(a) Total Pressure Ratio

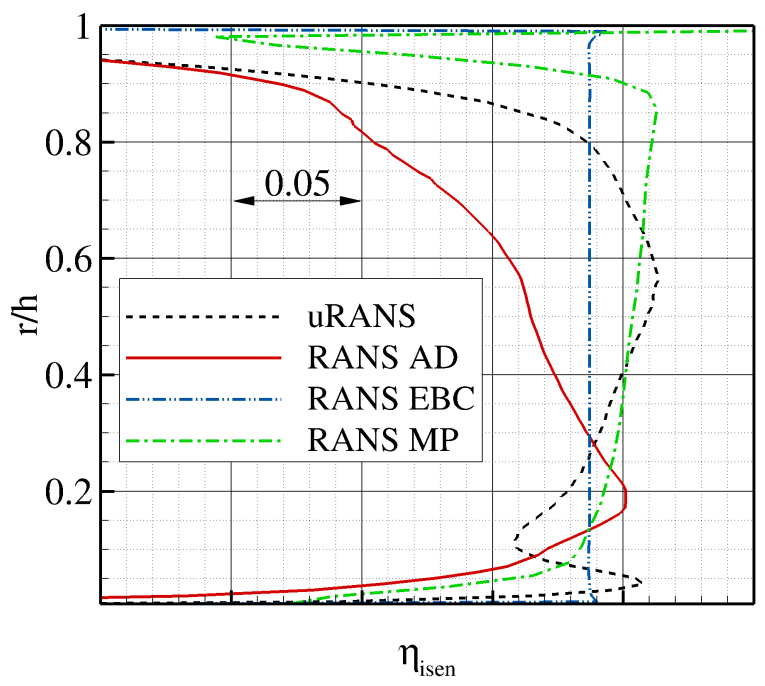

(b) Isentropic Efficiency

Fig. 14 Pitch averaged radial distributions in the bypass duct aft of the stator plane (S3) for the Take-Off operating point.

Table 5 Thrust component distribution comparison (TO).

\begin{tabular}{|l|c|c|}
\hline$\%$ Overall Thrust & uRANS & RANS Actuator Disk \\
\hline Rotor & $60 \%$ & $60 \%$ \\
\hline Stator & $28 \%$ & $27 \%$ \\
\hline Core & $12 \%$ & $14 \%$ \\
\hline
\end{tabular}

In Table 6 global engine performance metrics for the actuator disk approach and the engine boundary condition are compared against the uRANS results. As a reference the mixing-plane simulation data are shown as well. It can be observed that the actuator disk model is able to capture the engine massflow within $4 \%$ and fan pressure ratio within $3 \%$ of the uRANS results whereas deviations in terms of thrust are a bit larger at 7.6\%. This larger error in thrust was already observed for the ADP case. Isentropic efficiency is underestimated by $4.5 \%$ with the AD model. In comparison, the engine boundary condition results agree very well with the uRANS results for the massflow and FPR. The error in terms of thrust is of the same magnitude as with the AD model however in the positive direction resulting in higher thrust than the uRANS simulation. Isentropic efficiency is again overestimated with the engine boundary condition. Within the mixing-plane simulation the massflow is slightly higher compared to the uRANS computation whereas the FPR is almost identical. Isentropic efficiency estimate with the mixing-plane approach is around $1 \%$ higher than in the uRANS simulation. Overall the agreement between TRACE RANS mixing-plane and TAU uRANS results is again very good.

Table 6 Performance characteristics compared to uRANS data (TO).

\begin{tabular}{|l|c|c|c|}
\hline$\frac{X-X_{U R A N S}}{X_{U R A N S}}$ & RANS Actuator Disk & RANS Engine BC & RANS Mixing-Plane \\
\hline$\dot{m}$ & $-3.7 \%$ & $-1.0 \%$ & $+1.9 \%$ \\
\hline$F P R$ & $-2.9 \%$ & $-0.3 \%$ & $-0.1 \%$ \\
\hline$\eta_{i s e n}$ & $-4.5 \%$ & $+1.7 \%$ & $+1.2 \%$ \\
\hline$F_{T}$ & $-7.6 \%$ & $+7.2 \%$ & n/a \\
\hline
\end{tabular}




\section{Take-Off Sideslip}

The Take-Off sideslip case was used to test the engine modeling approaches for their ability to model engine performance with strongly disturbed inflow. Due to the high local incidence the flow separates at the inlet lip and a total pressure disturbance runs into the engine. Figure 15 depicts this phenomenon for the uRANS simulation, the AD model and the engine boundary condition (no TRACE data was available for this case). Values of negative $c f x$ are shown to illustrate the extent of the flow separation. The $c f x$-pattern for the engine boundary condition in Fig. $15 \mathrm{c}$ is almost symmetrical due to the fact that no rotation is imposed to the flow. This is not a realistic representation because the uRANS data and the AD simulation indicate that there is a significant upstream effect of the engine swirl. For both the uRANS and the AD data a deviation of the flow separation region in the direction of engine rotation can be observed. Nevertheless the extent of the flow separation for the AD model is significantly larger than for the uRANS simulation.

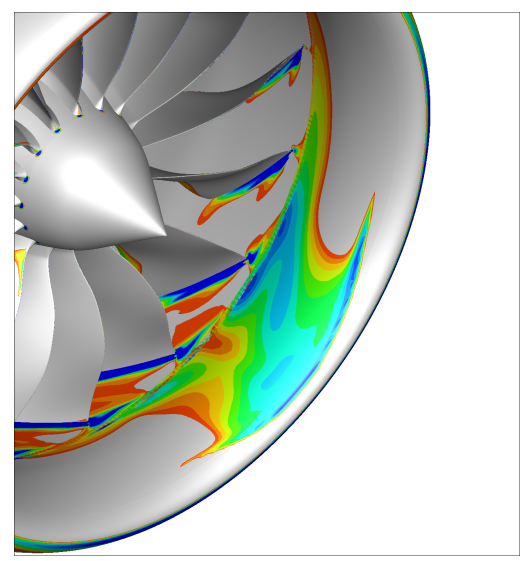

(a) URANS

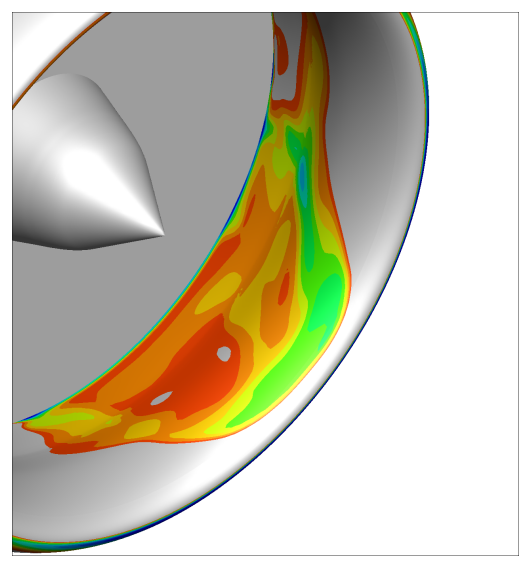

(b) RANS Actuator Disk

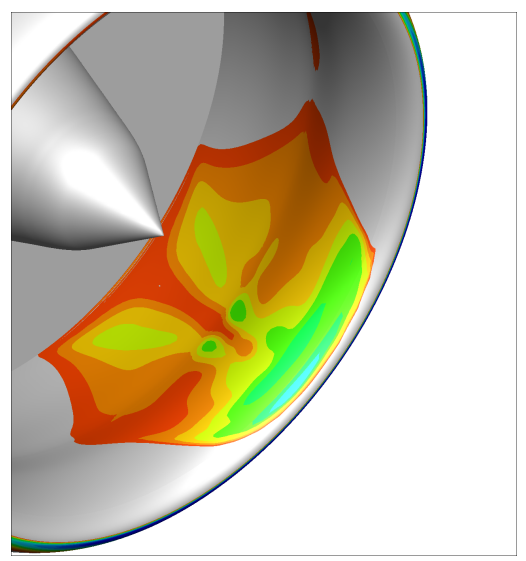

(c) RANS Engine BC

Fig. 15 Flow separation on inlet lip for different engine modeling methodologies visualized by contours of negative $c f x$ (TOSL).

The size of the disturbance flowing into the engine is varying for the different engine modeling approaches. Figure 16 shows the total pressure loss on the chimera plane in front of the rotor (S1). For the uRANS simulation in Fig. 16a additional spots of reduced total pressure aside from the primary perturbation can be seen. These result from the proximity of the S1 surface to the blades of the rotor. The overall size of the inflow disturbance is similar for all three methodologies. However for the AD model and the engine boundary condition it extends over a slightly larger circumferential region than for the uRANS data. This also applies to the extent in radial direction where the disturbance is smaller for the uRANS data compared to the AD model and the engine boundary condition.

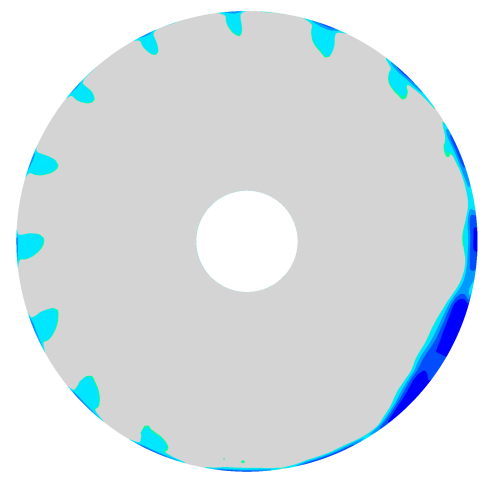

(a) URANS

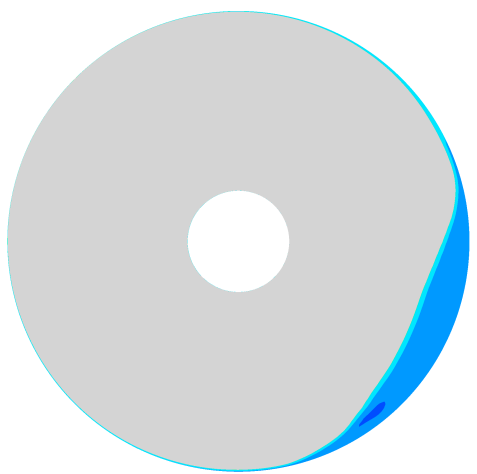

(b) RANS Actuator Disk

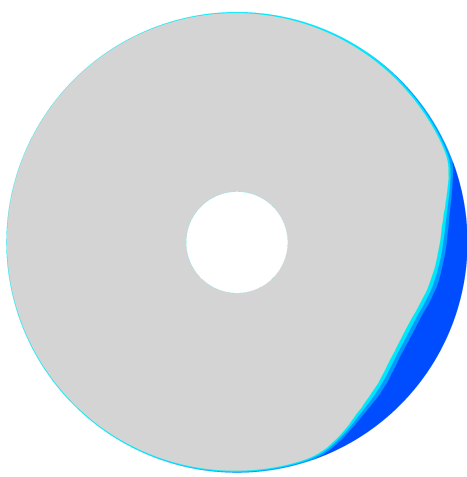

(c) RANS Engine BC

Fig. 16 Total pressure inlet distortion on chimera plane in front of the rotor block (S1) for the TOSL operating point; total pressure values larger than freestream total pressure are blanked. 
A more detailed analysis of the propagation of the perturbation through the engine was done for the uRANS and AD data in Fig. 17. Total pressure distributions at the stations S1, S2 and S3 are shown for both methodologies. The direction of fan rotation is counterclockwise. As already discussed the position and extent of the inflow distortion is modeled well by the actuator disk simulation (see S1 in Fig. 17p. Looking at position S2 behind the rotor the wakes of the rotor blades can be identified for the uRANS computation. In the uRANS simulation the region of total pressure deficit is reenergized and only small spots of lower total pressure remain. Behind the stator stage (S3) the remains of the disturbance can be identified as corner stall regions extending in circumferential direction by around $70^{\circ}$. The AD model is not able to reproduce this. The region of distorted flow is even enlarged by the actuator disk model in radial direction. When comparing the circumferential position of the distortion for all three positions it can be seen that in the uRANS computation the distortion has moved by around 90 degrees between position S1 and S3 whereas the movement with the AD model corresponds to an angle between 30 and 45 degrees. Nevertheless the general shape of the flow field in terms of maximum total pressure and minimum total pressure positions is reproduced by the AD model. Also the lower total pressure region at the hub 11 o'clock position at station $\mathrm{S} 2$ is predicted by the actuator disk simulation.

The component-wise thrust evaluation for this case can be seen in Table 7 for the uRANS and the AD approach. The thrust portion coming from the rotor and the core section in the AD simulation is higher compared to the uRANS data whereas the stator section delivers less thrust. It follows that the deficit in overall thrust in Table 8 mainly results from the stator stage.

Table 7 Thrust component distribution comparison (TOSL)

\begin{tabular}{|l|c|c|}
\hline$\%$ Overall Thrust & uRANS & RANS Actuator Disk \\
\hline Rotor & $60 \%$ & $62 \%$ \\
\hline Stator & $28 \%$ & $24 \%$ \\
\hline Core & $13 \%$ & $14 \%$ \\
\hline
\end{tabular}

Global engine performance was evaluated for this case as well. Table 8 shows the deviations between of the actuator disk and the engine boundary condition compared to the uRANS results. Massflow and FPR show a large deficit for the actuator disk model. This results from the inability of the AD model to reenergize the flow in the region of distorted inflow. As a result the total thrust is also largely underestimated by the model. The overall fan performance in terms of isentropic efficiency is also predicted to be worse than it actually is in the uRANS data. As expected the engine boundary condition correctly models the massflow since this was specified as a target value. Also the FPR matches the uRANS data very well. As a consequence of not adapting the total temperature ratio in the model the isentropic efficiency is unrealistically high. Actually it is significantly above unity, and thus nonphysical, for this case. Thrust prediction is in turn very good due to the correct reproduction of massflow and pressure ratio.

Table 8 Performance characteristics compared to uRANS data (TOSL)

\begin{tabular}{|l|c|c|}
\hline$\frac{X-X_{U R A N S}}{X_{U R A N S}}$ & RANS Actuator Disk & RANS Engine BC \\
\hline$\dot{m}$ & $-7.2 \%$ & $-0.9 \%$ \\
\hline$F P R$ & $-5.0 \%$ & $+0.4 \%$ \\
\hline$\eta_{i s e n}$ & $-11.5 \%$ & $+21.5 \%$ \\
\hline$F_{T}$ & $-12.9 \%$ & $+3.1 \%$ \\
\hline
\end{tabular}



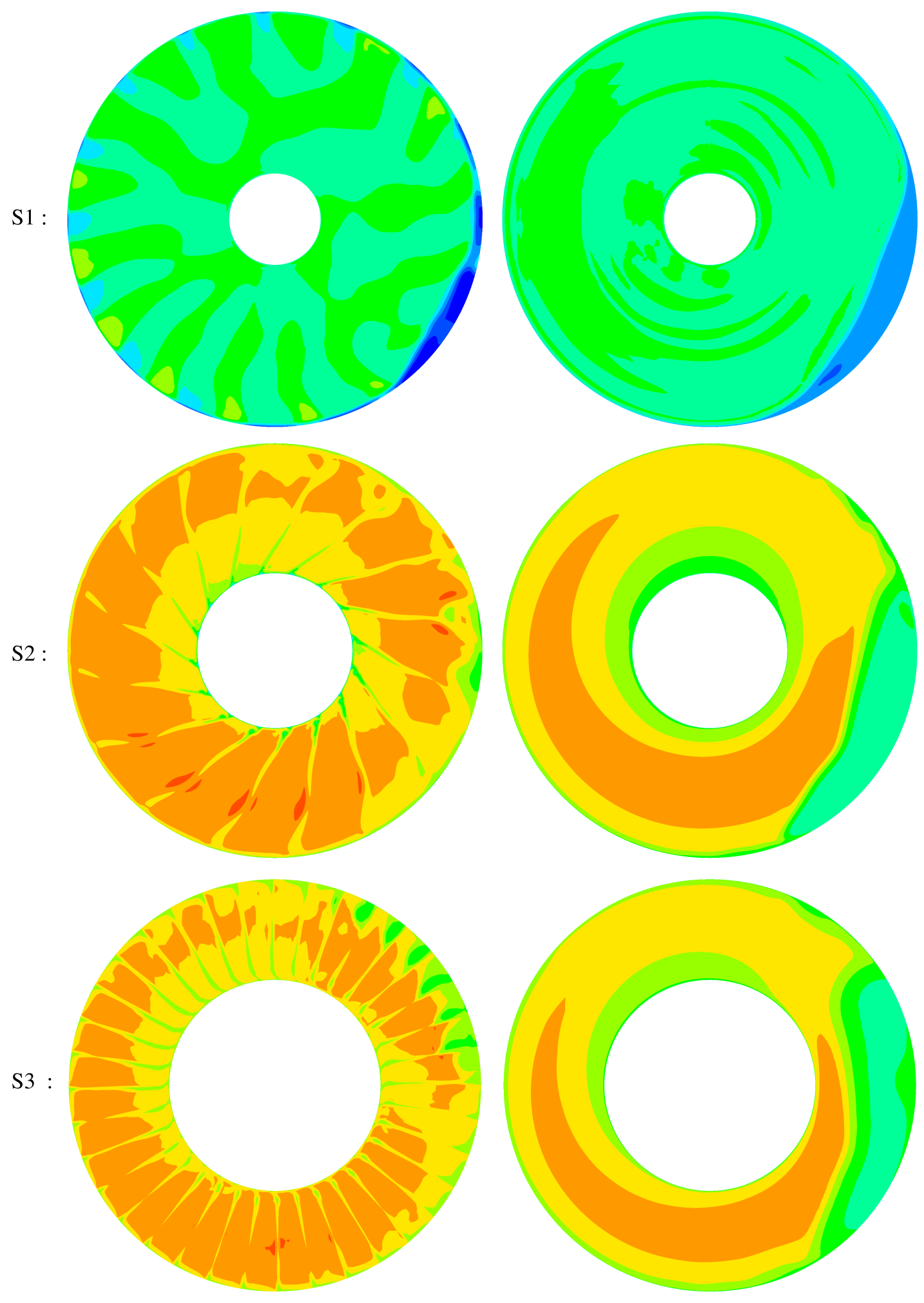

(a) URANS

(b) RANS Actuator Disk

Fig. 17 Total pressure distribution at stations S1, S2 and S3 for the TOSL operating point 


\section{Conclusion}

A novel approach of modeling turbofan engines in RANS simulations with AD boundary conditions based on BEMT was introduced for the DLR flow solver TAU. The approach was tested on an isolated UHBR engine under various conditions and compared to uRANS computations as well as steady computations with conventional engine boundary conditions. The results were also compared to results from steady simulations with mixing planes that were carried out with TRACE. The data of the TAU uRANS computation and the TRACE RANS mixing-plane simulation show very good agreement building confidence that results of both flow solvers are of high level of accuracy. This holds true for global engine performance results as well as the depicted radial distributions. Initial results from the AD approach indicate a generally good agreement with the ones of the uRANS computation while additional potential for improvement remains. The most important findings regarding the generation of $\mathrm{AD}$ model input data and the model performance are as follows:

The performance of the AD approach is highly dependent on the quality of the input data that reflect the blade properties. For the most part of the rotor/stator blades, sufficiently accurate sectional aerodynamic data can be derived via 2D RANS simulations when cascade effects and streamwise static pressure evolution are considered. The quality of the estimation decreases towards the blade tips, in particular of the fan blades, as 3D effects gain in importance. Modeling centrifugal effects may further improve the quality of the aerodynamic input data.

The AD model was able to accurately predict the global engine performance for the ADP and the Take-Off case. The distribution of thrust between the rotor and the stator section was reproduced correctly as well. The AD model proofed the ability to represent local blade response effects and predicted local swirl angles for the rotor and stator section. Despite its shortcoming in reproducing the 0D-results for the distorted inflow case, the AD model allowed for a more realistic estimate of the flow field inside the engine than the thermodynamic boundary condition. The additional computational effort compared to the conventional thermodynamic boundary condition was only $15 \%-25 \%$ although stability of the simulation was difficult to ensure in some cases. Overall the model showed the potential to be very useful for future engine airframe integration studies.

Future investigations will focus on the position and the form of the disk which are known to have an influence on the overall performance of the model. Furthermore the extraction of airfoil geometries for input data generation by conical surfaces instead of planar surfaces promises to improve the results. The shortcoming of providing the correct total pressure rise in the tip region indicates that additional 3D effects need to be accounted for inside the model. Issues leading to flow separation under certain circumstances might be addressed by a different formulation of the wall treatment on the disk.

\section{Acknowledgments}

The authors wish to thank A. Raichle for sharing his insights and for his continued support with the actuator disk method and A. Stuermer for initiating this research and for the extensive discussions on fan and propeller aerodynamics.

\section{References}

[1] Joo, W. G., and Hynes, T. P., "The Application of Actuator Disks to Calculations of the Flow in Turbofan Installations," Journal of Turbomachinery, Vol. 119, No. 4, 1997, pp. 733-741.

[2] Joo, W. G., and Hynes, T. P., "The Simulation of Turbomachinery Blade Rows in Asymmertric Flow Using Actuator Disks," Journal of Turbomachinery, Vol. 119, No. 4, 1997, pp. 723-732.

[3] Kim, S., Yang, S., Lee, D., Baftalovski, S., and Makarov, V., "Three-Dimensional Flow Calculation Around/Through Isolated Nacelle with an Actuator Disk Modeling," 35th AIAA/ASME/SAE/ASEE Joint Propulsion Conference and Exhibit, 1999.

[4] Burlot, A., Sartor, F., Vergez, M., Méheut, M., and Barrier, R., "Method Comparison for Fan Performance in Short Intake Nacelle," 2018 Applied Aerodynamics Conference, 2018.

[5] Schnell, R., Golfhahn, E., and Julian, M., "Design and Performance of a Low Fan-Pressure-Ratio Propulsion System," 25th International Symposium on Air Breathing Engines ISABE 2019, Canberra, Australia, 2019.

[6] Gerhold, T., "Overview of the Hybrid RANS Code TAU," MEGAFLOW - Numerical Flow Simulation for Aircraft Design, edited by N. Kroll and J. K. Fassbender, Springer Berlin Heidelberg, Berlin, Heidelberg, 2005, pp. 81-92. 
[7] Spalart, P., and Allmaras, S., “A one-equation turbulence model for aerodynamic flows," 30th Aerospace Sciences Meeting and Exhibit, 1992.

[8] Madrane, A., Heinrich, R., and Gerhold, T., "Implementation of the Chimera Method in the Unstructured Hybrid DLR Finitve Volume TAU-Code," 6th Overset Composite Grid and Solution Technology Symposium, Ft. Walton Beach, FL, USA, 2002, pp. 524-534.

[9] Stuermer, A., “Assessing Turbofan Modeling Approaches in the DLR TAU-Code for Aircraft Aerodynamics Investigations," AIAA Scitech 2019 Forum, 2019.

[10] Froude, R., "On the Part Played in Propulsion by Differences of Fluid Pressure," Transactions of the Institution of Naval Architects, Vol. 30, 1889, pp. 390-324.

[11] Rankine, W., "On the Mechanical Principles of the Action of Propellers," Transactions of the Institution of Naval Architects, Vol. 6, 1865, pp. 13-39.

[12] Raichle, A., "Flux Conservative Discretization of the Actuator Disk Model as a Discontinuity Surface," Ph.D. thesis, Technical University Braunschweig, 2017.

[13] Raichle, A., Melber-Wilkending, S., and Himisch, J., "A New Actuator Disk Model for the TAU Code and Application to a Sailplaine with a Folding Engine," New Results in Numerical and Experimental Fluid Mechanics VI, edited by C. Tropea, S. Jakirlic, H.-J. Heinemann, R. Henke, and H. Hönlinger, Springer Berlin Heidelberg, Berlin, Heidelberg, 2008 , pp. 52-61.

[14] Keller, D., and Rudnik, R., "Numerical investigations of aerodynamic properties of a proller blown circulation control system on a high wing aircraft," CEAS Aeronaut J, Vol. 7, 2016.

[15] Keller, D., and Rudnik, R., "Investigation and improvement of directional stability and control of a propeller-driven STOL aircraft," CEAS Aeronaut J, Vol. 10, 2019, pp. 909 - 924.

[16] Lenfers, C., Beck, N., and Bauer, M., "Propeller and active high lift wing interaction in experiment and simulation," New Results in Numerical and Experimental Fluid Mechanics, Vol. X, No. 132, 2016, pp. 51 - 61.

[17] Glauert, H., “Div. L Airplane Propellers,” Aerodynamic Theory, Vol. IV, edited by W. F. Durand, Julius Springer, Berlin, 1935.

[18] Schnell, R., and Corroyer, J., "Coupled Fan and Intake Design Optimization for Installed UHBR-Engines with Ultra-Short Nacelles," 23rd International Symposium on Air Breathing Engines ISABE, 2015.

[19] Schnell, R., Yin, J., Voss, C., and Nicke, E., "Assessment and Optimization of the Aerodynamic and Acoustic Characteristics of a Counter Rotating Open Rotor," ASME Journal of Turbomachinery, Vol. 134, 2012.

[20] Schönweitz, D., Theune, M., and Schnell, R., "Inlet Distortion Sensitivity of Fans with Different Pressure Ratios," 23rd International Symposium on Air Breathing Engines ISABE, Phoenix/AZ, USA, 2015.

[21] Schnell, R., Goldhahn, E., and Julian, M., "Design and Performance of a Low Fan-Pressure-Ratio Propulsion System," Proceedings of 25th International Symposium on Air Breathing Engines ISABE, Canberra, Australia, 2019.

[22] Meheut, M., Sartor, F., Vergez, M., Laban, M., and Schnell, A., R. amd Stürmer, "Assessment of Fan/Airframe aerodynamic performance using $360^{\circ}$ uRANS computations: Code-to-Code comparison between ONERA, DLR and NLR," AIAA SciTech Forum, 2019.

[23] Schnell, R., "Investigation of the Acoustic Nearfield of a Transsonic-Fanstage by Time-Domain CFD-Calculations with Arbitrary Balde Counts," ASME Turbo-Expo, 2004.

[24] Frey, C., Ashcroft, G., Kersken, H., Schönweitz, D., and Mennicken, M., "Simulation of Indexing and Clocking with Harmonic Balance," International Journal of Turbomachinery, Propulsion and Power, Vol. 3, No. 1, 2017. 